\title{
Z Transformation by Pascal Matrix and its Applications in the Design of IIR Filters
}

\author{
F. J. García-Ugalde*${ }^{* 1}$ B. Psenicka ${ }^{2}$, M.O. Jiménez-Salinas ${ }^{3}$ \\ 1,2,3 Universidad Nacional Autónoma de México. \\ Facultad de Ingeniería, División de Ingeniería Eléctrica \\ Circuito Exterior, Ciudad Universitaria, Coyoacán \\ 04510, Ciudad de México, Mexico. \\ *fgarciau@unam.mx
}

\begin{abstract}
In this work, we summarize a direct method to transform the low-pass continuous-time transfer function $H(s)$ to several discrete-time $H(z)$ transfer functions. Our algorithm uses the Pascal matrix that is built from the rows of a Pascal Triangle. The inverse transformation is obtained with the Pascal matrix without computing the determinant of the system, which simplifies the process to obtain the associated analog transfer function $H(s)$ if the discrete transfer function $H(z)$ is known. In addition, the algorithm is easy to program on a personal computer or scientific calculator because all the computations are made using matrices. The algorithm presented is illustrated with numerical examples.
\end{abstract}

Keywords: Analog filters, Digital filters, Pascal matrix, Bilinear Z-transform, IIR filters.

\section{RESUMEN}

En el presente trabajo, resumimos un método directo para transformar la función de transferencia paso bajas del tiempo continuo $H(s)$ a diferentes funciones de transferencia del tiempo discreto $H(z)$. Nuestro algoritmo utiliza la matriz de Pascal que se construye con los renglones del triángulo de Pascal. La transformación inversa se obtiene con la matriz de Pascal sin calcular el determinante del sistema, lo que simplifica el proceso para obtener la correspondiente función de transferencia analógica $H(s)$ si la función de transferencia discreta $H(z)$ es conocida. Además, el algoritmo es fácil de programar en una computadora personal o una calculadora científica, debido a que todos los cálculos se realizan con matrices. El algoritmo presentado es ilustrado con ejemplos numéricos.

\section{Introduction}

In the context of the digital filter design, a great deal of research has been done to facilitate their computation. As a matter of fact, the Pascal matrix defined in [1] has proved its utility in this field. In the literature [2], the s-z transformation is done in two steps. First, the frequency transformation in the $s$ domain is done, then the Pascal matrix for the bilinear $z$ transformation is used to obtain the associated digital filter. We shall give in this work a direct method to transform a filter from the continuous time to the discrete time.

The continuous-time circuit of a filter is completely described by the transfer function given in (1):

$$
H(s)=\frac{A_{0}+A_{1} s+A_{2} s^{2}+\cdots+A_{N} s^{N}}{B_{0}+B_{1} s+B_{2} s^{2}+\cdots+B_{N} s^{N}}=\frac{\sum_{i=0}^{N} A_{i} s^{i}}{\sum_{i=0}^{N} B_{i} s^{i}}
$$


where the coefficients $A_{i}$ and $B_{i}$ are real. Those coefficients can be represented in vector form, as follows:

$$
\boldsymbol{A}=\left(A_{0}+A_{1}+A_{2}+\cdots+A_{N}\right) \quad \boldsymbol{B}=\left(B_{0}+B_{1}+B_{2}+\cdots+B_{N}\right)
$$

In a similar way, the discrete filter can be characterized with the transfer function $H(z)$ given in (3), which also has real coefficients $a_{i}$ and $b_{i}$ that are obtained using the bilinear $z$-transformation.

$$
\begin{gathered}
H(z)=\frac{a_{0}+a_{1} z^{-1}+a_{2} z^{-2}+\cdots+a_{N} z^{-N}}{b_{0}+b_{1} z^{-1}+b_{2} z^{-2}+\cdots+b_{N} z^{-N}}=\frac{\sum_{i=0}^{N} a_{i} z^{-i}}{\sum_{i=0}^{N} b_{i} z^{-i}} \\
\boldsymbol{a}=\left(a_{0}+a_{1}+a_{2}+\cdots+a_{N}\right) \quad \boldsymbol{b}=\left(b_{0}+b_{1}+b_{2}+\cdots+b_{N}\right)
\end{gathered}
$$

We will now give a brief explanation of the Pascal matrix, if in Equation (1) we substitute the relationship between $s-z$ of bilinear transform $\left(s=c \frac{1-z^{-1}}{1+z^{-1}}\right)$, then we obtained (5)

$$
\begin{gathered}
H(z)=\frac{\sum_{i=0}^{N} A_{i} c^{i}\left[\frac{1-z^{-1}}{1+z^{-1}}\right]^{i}}{\sum_{i=0}^{N} B_{i} c^{i}\left[\frac{1-z^{-1}}{1+z^{-1}}\right]^{i}} \\
H(z)=\frac{\sum_{i=0}^{N} A_{i} c^{i}\left(1-z^{-1}\right)^{i}\left(1+z^{-1}\right)^{N-i}}{\sum_{i=0}^{N} B_{i} c^{i}\left(1-z^{-1}\right)^{i}\left(1+z^{-1}\right)^{N-i}}
\end{gathered}
$$

Using the binomial theorem

$(a+b)^{n}=\sum_{k=0}^{n}\left(\begin{array}{l}n \\ k\end{array}\right) a^{n-k} b^{k}$

where $\left(\begin{array}{l}n \\ k\end{array}\right)=\frac{n !}{n !(n-k) !}$, we can distribute the numerator of (5) as follows:

$$
\begin{aligned}
& \sum_{i=0}^{N} A_{i} c^{i}\left(1-z^{-1}\right)^{i}\left(1+z^{-1}\right)^{N-i} \\
& =\sum_{i=0}^{N} A_{i} c^{i}\left[\sum_{k=0}^{i}\left(\begin{array}{l}
i \\
k
\end{array}\right)(-1)^{k} z^{-k} \sum_{k=0}^{N-i}\left(\begin{array}{c}
N-i \\
k
\end{array}\right) z^{-k}\right]
\end{aligned}
$$

The terms in brackets can be expressed as a matrix of size $(N+1) \times(N+1)$, as shown in [3]. This matrix is called the Pascal matrix $P$ and, it is very useful in the $s-z$ transformation. A relationship between (7) and (3) can be found rearranging and changing some indices, as in [3].

$\sum_{i=0}^{N} a^{i} z^{-i}=\sum_{i=0}^{N} A_{i} c^{i}\left[P_{i, j}\right] z^{-i}$

then the Pascal matrix $P_{i, j}$ is defined as $[3,4]$.

$$
\begin{gathered}
P_{i, j}=\sum_{n=0}^{i}\left(\begin{array}{c}
N-j \\
n
\end{array}\right)\left(\begin{array}{c}
j \\
i-n
\end{array}\right)(-1)^{i-n} \\
i, j=0,1, \ldots, N
\end{gathered}
$$

where $i$ and $j$ are the indices of the row and column of the Pascal matrix, respectively.

The coefficients of the numerator of the digital filter $\boldsymbol{a}$ can be computed using (10). The coefficients $b_{i}$ of the denominator can be obtained in a similar way as the coefficientes of the numerator $a_{i}$ as shown in (11)

$$
\begin{aligned}
& {\left[a_{i}\right]=\left[P_{i, j}\right]\left[A_{i} c^{i}\right]} \\
& {\left[b_{i}\right]=\left[P_{i, j}\right]\left[B_{i} c^{i}\right]}
\end{aligned}
$$




\section{Lowpass to Low-pass Transformation}

The discrete low-pass filter is obtained using the bilinear $z$-transformation from (12),

$$
s=c \frac{1-z^{-1}}{1+z^{-1}}, \quad c=\cot \left(\frac{\pi f_{1}}{f_{s}}\right)
$$

where $f_{1}$ is the cut-off frequency of the low-pass filter and $f_{s}$ is the sampling frequency. In order to obtain the coefficients $\boldsymbol{a}$ and $\boldsymbol{b}$ from the vectors $\boldsymbol{A}$ and $\boldsymbol{B}$, we compare the numerators and denominators of the transfer functions (1) and (3). For example, if we substitute (12) in (1) for $N=2$, we obtain the following expression:

$$
H(z)=\frac{A_{0}+A_{1} c+A_{2} c^{2}+z^{-1}\left(2 A_{0}-2 A_{2} c^{2}\right)+z^{-2}\left(A_{0}-A_{1} c+A_{2} c^{2}\right)}{B_{0}+B_{1} c+B_{2} c^{2}+z^{-1}\left(2 B_{0}-2 B_{2} c^{2}\right)+z^{-2}\left(B_{0}-B_{1} c+B_{2} c^{2}\right)}
$$

Comparing the coefficients in (13) with the same exponents of the variable $z$, we get the matrix equation:

$$
\left[\begin{array}{l}
a_{0} \\
a_{1} \\
a_{2}
\end{array}\right]=\left[\begin{array}{ccc}
1 & 1 & 1 \\
2 & 0 & -2 \\
1 & -1 & 1
\end{array}\right] \cdot\left[\begin{array}{l}
A_{0} \\
A_{1} \\
A_{2}
\end{array}\right]=\boldsymbol{P}_{\boldsymbol{L P}} \cdot \boldsymbol{A}^{(i)}
$$

A similar equation can be obtained for vector $\boldsymbol{b}$. These equations can be represented in the following compact form:

$$
\begin{aligned}
& \boldsymbol{a}=\boldsymbol{P}_{\boldsymbol{L P}} \cdot \boldsymbol{A}^{(i)} \\
& \boldsymbol{b}=\boldsymbol{P}_{\boldsymbol{L P}} \cdot \boldsymbol{B}^{(i)}
\end{aligned}
$$

Where $\boldsymbol{P}_{\boldsymbol{L P}}$ is the low-pass Pascal matrix, and $\boldsymbol{A}^{(i)}$ and $\boldsymbol{B}^{(i)}$ are the vectors represented by

$$
\begin{aligned}
& \boldsymbol{A}^{(i)}=\left(A_{0}, A_{1} c, A_{2} c^{2}, \ldots, A_{N} c^{N}\right) \\
& \boldsymbol{B}^{(i)}=\left(B_{0}, B_{1} c, B_{2} c^{2}, \ldots, B_{N} c^{N}\right) \\
& 1 \quad n_{0} \\
& 111 n_{1}
\end{aligned}
$$

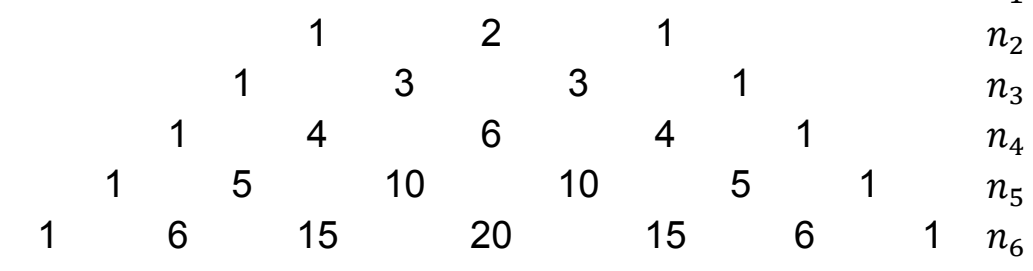




\section{Z Transformation by Pascal Matrix and its Applications in the Design of IIR Filters, F. J. García-Ugalde et al. / 355-366}

From the classical Pascal triangle in (17), we can observe that the elements of the base $n_{2}$ form the last column of the Pascal matrix in (14) with the exception that the even rows are negative and the first column of the same equation corresponds to the third row of the Pascal triangle. We conclude that the Pascal matrix can be formed taking into account the following considerations:

1. All the elements in the first row of the Pascal Matrix must be ones.

2. The elements in the last column can be computed by the following expression:

$$
P_{i, n+1}=(-1)^{i-1} \frac{N !}{(N+1-i) !(i-1) !} \quad i=1, \ldots, N+1
$$

and the remaining elements in the Pascal matrix can be computed with the following expression:

$$
P_{i, j}=P_{i-1, j}+P_{i-1, j+1}+P_{i, j+1} \text { with } i, j=2,3, \ldots, N, N+1
$$

\subsection{Example}

In this example, we wish to transform the low-pass transfer function $H_{l p}(s)$ to its discrete low-pass version $H_{l p}(z)$ using the following parameters:

$$
f_{s}=8000[\mathrm{~Hz}], f_{1}=2200[\mathrm{~Hz}], H_{l p}(s)=\frac{s^{2}+5.153}{0.929 s^{3}+2.781 s^{2}+4.344 s+5.153}
$$

First, we calculate the constant $c$ of the bilinear $z$ transformation using (12):

$$
c=\cot \left(\frac{\pi \cdot 2200}{8000}\right)=0.8540806
$$

Using the Pascal matrix $\left(\boldsymbol{P}_{L P}\right)$ for order $N=3$, the numerator and denominator of the transfer function $H_{l p}(z)$ are computed as follows:

$$
\begin{gathered}
{\left[\begin{array}{l}
a_{0} \\
a_{1} \\
a_{2} \\
a_{3}
\end{array}\right]=\left[\begin{array}{cccc}
1 & 1 & 1 & 1 \\
3 & 1 & -1 & -3 \\
3 & -1 & -1 & 3 \\
1 & -1 & 1 & -1
\end{array}\right] \cdot\left[\begin{array}{c}
5.153 \\
0 \\
0.854^{2} \\
0
\end{array}\right]=\left[\begin{array}{c}
5.8823 \\
14.7297 \\
14.7297 \\
5.8823
\end{array}\right]} \\
{\left[\begin{array}{l}
b_{0} \\
b_{1} \\
b_{2} \\
b_{3}
\end{array}\right]=\left[\begin{array}{cccc}
1 & 1 & 1 & 1 \\
3 & 1 & -1 & -3 \\
3 & -1 & -1 & 3 \\
1 & -1 & 1 & -1
\end{array}\right] \cdot\left[\begin{array}{c}
5.153 \\
4.344 \cdot 0.854 \\
2.781 \cdot 0.854^{2} \\
0.929 \cdot .0 .854^{3}
\end{array}\right]=\left[\begin{array}{c}
11.4696 \\
15.4047 \\
11.4568 \\
2.8928
\end{array}\right]} \\
H_{l p}(z)=\frac{5.8823+14.7297 z^{-1}+14.7297 z^{-2}+5.8823 z^{-3}}{11.4696+15.4047 z^{-1}+11.4568 z^{-2}+2.8928 z^{-3}}
\end{gathered}
$$




\section{Low-pass to High-pass Transformation}

The low-pass to high-pass transformation is similar to the lowpass to low-pass case; first we substitute the variable $1 / s$ instead of $s$ in (18), then

$s=k \frac{z+1}{z-1}, \quad k=\tan \left(\frac{\pi f_{-1}}{f_{s}}\right)$

in the relation (24), $f_{-1}$ represents the cut-off frequency of the high-pass filter and $f_{s}$, the sampling frequency. If we substitute (24) in (1) and compare the numerator, for $N=3$, we get the following expressions in the matrix form:

$\boldsymbol{a}=\boldsymbol{P}_{\boldsymbol{H P}} \cdot \boldsymbol{A}^{(i i)} \quad \boldsymbol{b}=\boldsymbol{P}_{\boldsymbol{H P}} \cdot \boldsymbol{B}^{(i i)}$

$$
P_{i, j}=P_{i, j-1}+P_{i-1, j-1}+P_{i-1, j} \quad \text { with } i, j=2,3, \ldots, N+1
$$

The matrix $\boldsymbol{P}_{H \boldsymbol{P}}$ can also be obtained by flipping the columns of the Pascal matrix $\boldsymbol{P}_{\boldsymbol{L} \boldsymbol{P}}$ in the left-to-right direction [5]. The vectors $\boldsymbol{A}^{(i i)}$ and $\boldsymbol{B}^{(i i)}$ are represented in a similar way than $\boldsymbol{A}^{(i)}$ and $\boldsymbol{B}^{(i)}$,

$$
\begin{aligned}
& \boldsymbol{A}^{(i i)}=\left(A_{0}, A_{1} k, A_{2} k^{2}, \ldots, A_{N} k^{N}\right) \\
& \boldsymbol{B}^{(i i)}=\left(B_{0}, B_{1} k, B_{2} k^{2}, \ldots, B_{N} k^{N}\right)
\end{aligned}
$$

\subsection{Example}

In this example, we shall transform the low-pass transfer function $H_{l p}(s)$ to the high-pass transfer function $H_{h p}(z)$ using the following parameters:

$f_{s}=8000[\mathrm{~Hz}], f_{-1}=2200[\mathrm{~Hz}], H_{l p}(s)=\frac{s^{2}+5.153}{0.929 s^{3}+2.781 s^{2}+4.344 s+5.153}$

First, we compute the constant $k$ of the bilinear $z$ transform:

$$
k=\tan \left(\frac{\pi \cdot 2200}{8000}\right)=1.1708
$$

then, we use the expressions $\boldsymbol{a}=\boldsymbol{P}_{\boldsymbol{H P}} \cdot \boldsymbol{A}^{(i i)}$ and $\boldsymbol{b}=\boldsymbol{P}_{\boldsymbol{H P}} \cdot \boldsymbol{B}^{(i i)}$ for order $N=3$ to finally get the discrete transfer function $H_{h p}(z)$ :

$H_{h p}(z)=\frac{6.5238-14.0882 z^{-1}+14.0882 z^{-2}-6.5238 z^{-3}}{15.5420-12.2600 z^{-1}+11.0338 z^{-2}-2.3882 z^{-3}}$ 


\section{Low-pass to Band-pass Transformation}

The procedure considered in this section shows how to obtain a discrete band-pass filter characterized by the continuous-time transfer function $H_{b p}(s)$ from (1), using the $s-z$ transformation. The bandpass can be seen as a superposition of a low-pass filter and high-pass filter [6], [7]. Then the $s-z$ transform has the form:

$$
s=m\left(c \frac{z-1}{z+1}+k \frac{z+1}{z-1}\right) c=\cot \left(\frac{\pi f_{1}}{f_{s}}\right) k=\tan \left(\frac{\pi f_{-1}}{f_{s}}\right) m=\frac{\cot \left(\frac{\pi\left(f_{1}-f_{-1}\right)}{f_{s}}\right)}{c+k}
$$

In the last equation, $f_{s}$ represents the sampling frequency, $f_{-1}$ is the lower cut-off frequency and $f_{1}$ is the upper cut-off frequency of the band-pass filter. In order to obtain the coefficients $a_{i}$ and $b_{i}$ with $(i=0,1, \ldots, 2 N)$, we need to know the coefficients of the continuous vector $\boldsymbol{A}$ and $\boldsymbol{B}$, respectively. Then, we substitute (33) in (1), after that, we compare the numerator and the denominator of the resulting function with the transfer function in (3). For simplicity, and without loss of generalization, we choose as an example the order of the filter for $N=3$. Due to the low-pass to band-pass transformation, the transfer function $H_{b p}(z)$, becomes of order $N=6$. After comparing the numerators and denominators of the discrete and continuous transfer functions, we get the expression in matrix form:

$$
\left[\begin{array}{l}
a_{0} \\
a_{1} \\
a_{2} \\
a_{3} \\
a_{4} \\
a_{5} \\
a_{6}
\end{array}\right]=\left[\begin{array}{ccccccc}
1 & 1 & 1 & 1 & 1 & 1 & 1 \\
-6 & -4 & -2 & 0 & 2 & 4 & 6 \\
15 & 5 & -1 & -3 & -1 & 5 & 15 \\
-20 & 0 & 4 & 0 & -4 & 0 & 20 \\
15 & -5 & -1 & 3 & -1 & -5 & -15 \\
-6 & 4 & -2 & 0 & 2 & -4 & 6 \\
1 & -1 & 1 & -1 & 1 & -1 & 1
\end{array}\right] \cdot\left[\begin{array}{c}
A_{3} c^{3} m^{3} \\
A_{2} c^{2} m^{2} \\
A_{1} c m+3 A_{3} c^{2} \mathrm{~km}^{3} \\
A_{0}+2 A_{2} k c m^{2} \\
A_{1} k m+3 A_{3} k^{2} \mathrm{~cm}^{3} \\
A_{2} k^{2} m^{2} \\
A_{2} k^{3} m^{3}
\end{array}\right]=\boldsymbol{P}_{\boldsymbol{B P}} \cdot \boldsymbol{A}^{(i i i)}
$$

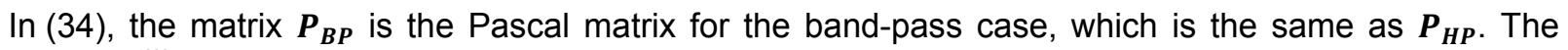
vector $\boldsymbol{A}^{(i i i)}$ is obtained as a recurrence. Unlike [8], we added the term $m$ in the recurrence to adjust the band of the filter; then $\boldsymbol{A}^{(i i i)}$ is obtained according to (35), see [8] pp. 878.

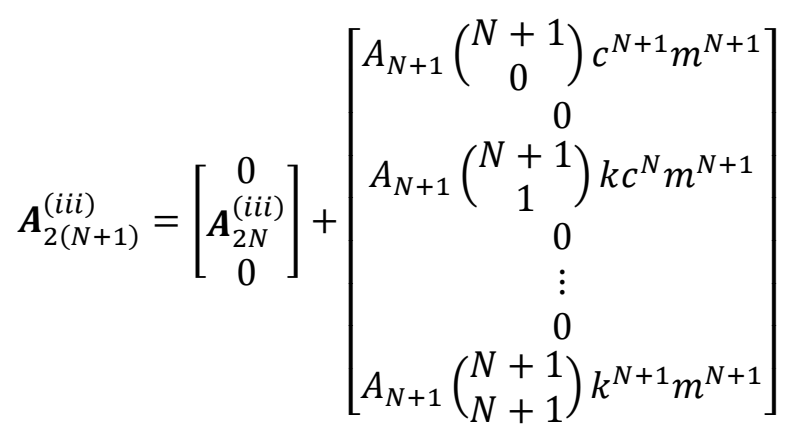

where $\boldsymbol{A}_{0}^{(i i i)}=\left[\boldsymbol{A}_{0}\right]$. The vector $\boldsymbol{B}^{(i i i)}$ is obtained in a similar way. 


\subsection{Example}

In this example, we shall transform a Chebychev low-pass filter to a band-pass filter with the following specifications, $a_{\max }=3[\mathrm{~dB}]$, order $N=4, f_{1}=3000[\mathrm{~Hz}], f_{-1}=1000[\mathrm{~Hz}], f_{s}=8000[\mathrm{~Hz}]$; using these parameters, we get the transfer function:

$$
H_{l p}(s)=\frac{0.1253}{s^{4}+0.5851 s^{3}+1.1692 s^{2}+0.4048 s+0.1770}
$$

First, we compute the constants $k, c, m$ :

$$
\begin{gathered}
k=\tan \left(\frac{\pi 1000}{8000}\right)=0.4142 c=\cot \left(\frac{\pi 3000}{8000}\right)=0.4142 \\
m=\frac{\cot \left(\frac{\pi+1000)}{8000}\right)}{0.4142+0.4142}=1.2071
\end{gathered}
$$

After that, we calculate the band-pass Pascal matrix and substitute $k, c$, and $m$ in vectors $\boldsymbol{A}^{(i i i)}$ and $\boldsymbol{B}^{(i i i)}$ for $N=8$ performing the following multiplications:

$$
\boldsymbol{a}=\boldsymbol{P}_{\boldsymbol{B P}} \cdot \boldsymbol{A}^{(i i i)} \quad \boldsymbol{b}=\boldsymbol{P}_{\boldsymbol{B P}} \cdot \boldsymbol{B}^{(i i i)}
$$

we get the coefficients of the numerator and denominator of the transfer function $H_{b p}(z)$, which is given in (40):

$H_{b p}(z)=\frac{0.1253-0.5012 z^{-2}+0.7518 z^{-4}-0.5012 z^{-6}+0.1253 z^{-8}}{3.3325+3.6528 z^{-2}+4.7236 z^{-4}+2.9384 z^{-6}+1.3563 z^{-8}}$

The coefficients multiplied by $z^{-(2 N-1)}$ are missing in the transfer function $H_{b p}(z)$ because the constants $c$ and $k$ are equal. The magnitude of the band-pass filter in (40) is shown in Figure 1.

\section{Low-pass to Band-stop Transformation}

To transform the continuous low-pass transfer function to the digital band-stop transfer function, we must use the $s-z$ transformation in the form:

$$
s=\frac{1}{m\left(c \frac{z-1}{z+1}+k \frac{z+1}{z-1}\right)}
$$




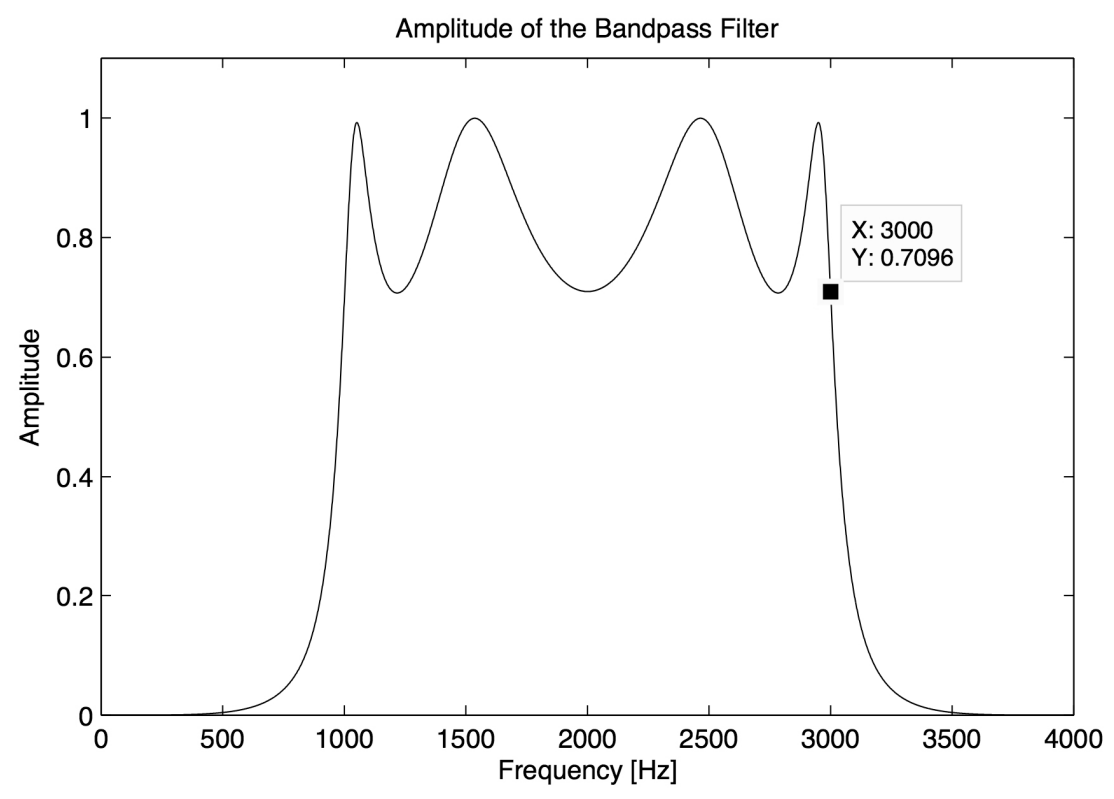

Figure 1. Magnitude of the band-pass filter.

It is easy to prove that the vector $\boldsymbol{A}^{(i v)}$ in (42) can be obtained reversing the coefficients $\left(A_{i}=A_{N-i}\right)$ of the vector $\boldsymbol{A}^{(i i i)}$ and the same for vector $\boldsymbol{B}^{(i v)}$. We do not need to do any change in the Pascal matrix, that is, the Pascal matrix for the band-pass case is exactly the same for the band-stop case $\left(\boldsymbol{P}_{\boldsymbol{B} \boldsymbol{P}}=\right.$ $\left.\boldsymbol{P}_{\boldsymbol{B} S}\right)$. Then the transformation is given by the following relations:

\subsection{Example}

$$
\boldsymbol{a}=\boldsymbol{P}_{B S} \cdot \boldsymbol{A}^{(i v)} \quad \boldsymbol{b}=\boldsymbol{P}_{B S} \cdot \boldsymbol{B}^{(i v)}
$$

In this example, we wish to transform the Chebychev low-pass transfer function $H_{l p}(z)$ to the band-stop transfer function $H_{b s}(z)$ using the following parameters $a_{\text {max }}=3[\mathrm{~dB}]$, order $N=4, f_{1}=3000[\mathrm{~Hz}$, $f_{-1}=1000[\mathrm{~Hz}], f_{s}=8000[\mathrm{~Hz}]$.

$$
H_{l p}(s)=\frac{0.1253}{s^{4}+0.5851 s^{3}+1.1692 s^{2}+0.4048 s+9.1770}
$$

First, we compute the parameters $k, c$, and $m$ and then we substitute them along with the coefficients $A_{i}$ and $B_{i}$ of the transfer function (43) in (42). After performing these matrix operations, we get the transfer function of the band-stop filter (44):

$$
H_{b s}(z)=\frac{0.1253+0.5012 z^{-2}+0.7518 z^{-4}+0.5012 z^{-6}+0.1253 z^{-8}}{3.3359-3.6528 z^{-2}+4.7236 z^{-4}-2.9315 z^{-6}+1.3563 z^{-8}}
$$


The digital transfer functions of the band-pass and band-stop filters are similar and they only differ in signs. The magnitude of the band-stop filter is shown in Figure 2.

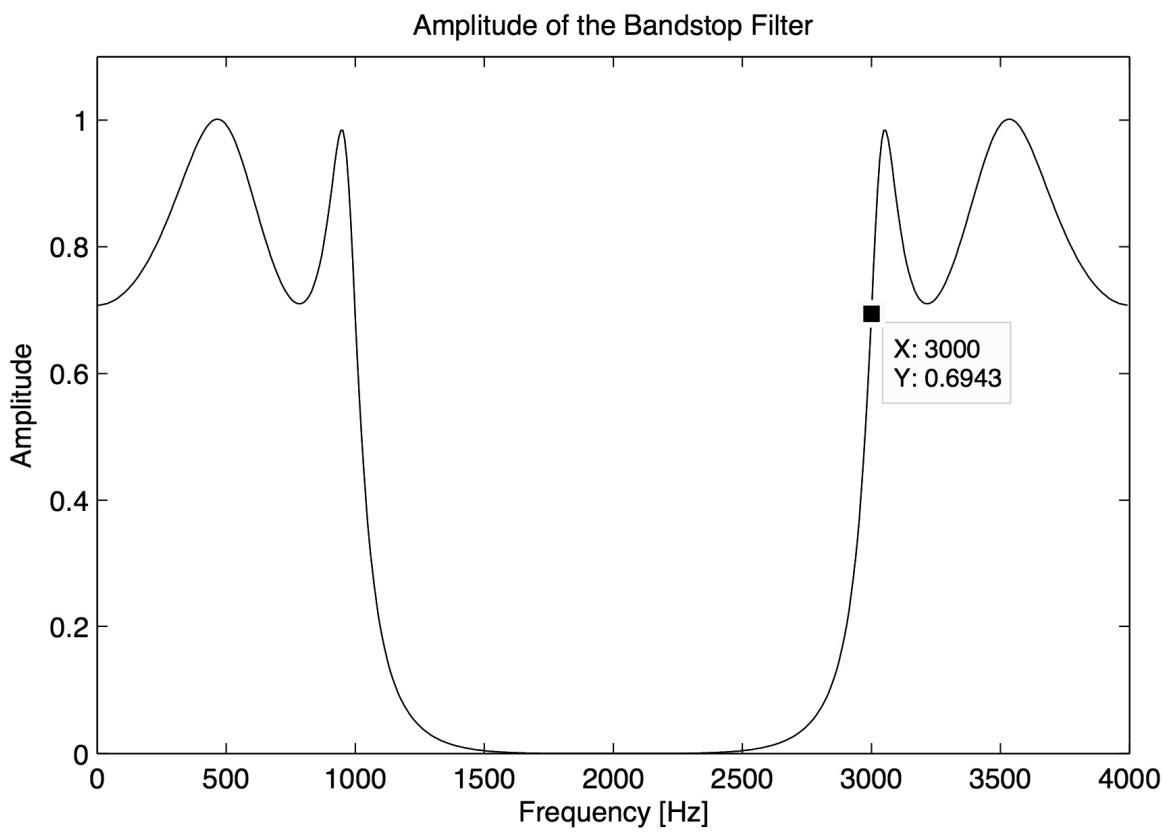

Figure 2. Magnitude of the band-stop filter.

\section{Inverse Transformation from z Domain to s Domain}

One of the properties of the Pascal matrix is that if we multiply a matrix by itself, we almost reach the identity matrix up to a scaling factor [5]. That means that if we wish to calculate the inverse of a Pascal matrix, we only need to normalize the matrix up to a scaling factor depending on its order to get its inverse. For instance, if we multiply the low-pass Pascal matrices $P_{4} \cdot P_{4}$, we obtain

That means that

$$
\begin{aligned}
& P_{4} \cdot P_{4}=\left[\begin{array}{ccccc}
1 & 1 & 1 & 1 & 1 \\
4 & 2 & 0 & -2 & -4 \\
6 & 0 & -2 & 0 & 6 \\
4 & -2 & 0 & 2 & -4 \\
1 & -1 & 1 & -1 & 1
\end{array}\right] \cdot\left[\begin{array}{ccccc}
1 & 1 & 1 & 1 & 1 \\
4 & 2 & 0 & -2 & -4 \\
6 & 0 & -2 & 0 & 6 \\
4 & -2 & 0 & 2 & -4 \\
1 & -1 & 1 & -1 & 1
\end{array}\right] \\
& =\left[\begin{array}{ccccc}
16 & 0 & 0 & 0 & 0 \\
0 & 16 & 0 & 0 & 0 \\
0 & 0 & 16 & 0 & 0 \\
0 & 0 & 0 & 16 & 0 \\
0 & 0 & 0 & 0 & 16
\end{array}\right] \\
& P_{4}^{-1}=\frac{1}{2^{4}} P_{4}
\end{aligned}
$$




\section{Z Transformation by Pascal Matrix and its Applications in the Design of IIR Filters, F. J. García-Ugalde et al. / 355-366}

and in the general case

$$
P_{N}^{-1}=\frac{1}{2^{N}} P_{N}
$$

where $N$ represents the order of the matrix. Then, the inverse of a Pascal matrix is very easy to calculate and, therefore, it is not necessary to calculate the determinant of the system; this simplifies the calculation of the transfer function in the $s$ domain if the transfer function in the $z$ domain is known. As an example, we shall transform the low-pass transfer function $H_{l p}(z)$ to the transfer function $H_{l p}(s)$. Let $H_{l p}(z)$ be the transfer function of the discrete system that works at a cut-off frequency $f_{1}=2000$ $[\mathrm{Hz}]$ and sampling frequency $f_{s}=8000[\mathrm{~Hz}]$.

$$
H_{l p}(z)=\frac{1+4 z^{-1}+6 z^{-2}+4 z^{-3}+z^{-4}}{10.6464+5.1716 z^{-2}+0.1880 z^{-4}}
$$

The parameter $c$ of the bilinear transformation according to (12) has the value

$$
c=\cot \left(\frac{\pi \cdot 2000}{8000}\right)=1.0
$$

using the Pascal matrix for $N=4$, we get

$$
\begin{gathered}
{\left[\begin{array}{c}
A_{0} \\
A_{1} c \\
A_{2} c^{2} \\
A_{3} c^{3} \\
A_{4} c^{4}
\end{array}\right]=\frac{1}{2^{4}}\left[\begin{array}{ccccc}
1 & 1 & 1 & 1 & 1 \\
4 & 2 & 0 & -2 & -4 \\
6 & 0 & -2 & 0 & 6 \\
4 & -2 & 0 & 2 & -4 \\
1 & -1 & 1 & -1 & 1
\end{array}\right] \cdot\left[\begin{array}{l}
1 \\
4 \\
6 \\
4 \\
1
\end{array}\right]=\left[\begin{array}{l}
1 \\
0 \\
0 \\
0 \\
0
\end{array}\right]} \\
{\left[\begin{array}{c}
B_{0} \\
B_{1} c \\
B_{2} c^{2} \\
B_{3} c^{3} \\
B_{4} c^{4}
\end{array}\right]=\frac{1}{2^{4}}\left[\begin{array}{ccccc}
1 & 1 & 1 & 1 & 1 \\
4 & 2 & 0 & -2 & -4 \\
6 & 0 & -2 & 0 & 6 \\
4 & -2 & 0 & 2 & -4 \\
1 & -1 & 1 & -1 & 1
\end{array}\right] \cdot\left[\begin{array}{c}
10.6464 \\
0 \\
5.1716 \\
0 \\
0.1880
\end{array}\right]=\left[\begin{array}{c}
1 \\
2.6131 \\
3.4142 \\
2.6131 \\
1
\end{array}\right]}
\end{gathered}
$$

then the transfer function of the analog low-pass filter is a Butterworth low-pass filter of order $n=4$. Constants $c, k$ and $m$ are used to avoid the distortions that are introduced by bilinear transformation.

$$
H_{l p}(s)=\frac{1}{s^{4}+2.6131 s^{3}+3.4142 s^{2}+2.6131 s^{2}+1}
$$




\section{Conclusions}

In this work we have used the Pascal matrix to transform the analog low-pass transfer function to the discrete transfer functions of the low-pass, high-pass, band-pass and band-stop filters. This algorithm is very simple for both the direct and inverse transformation. In addition, this method is easy to program due to the fact that all the operations imply the multiplication of matrices, even the inverse transformation does not need the calculation of the determinant of the matrix system. Based on the examples, the procedure has demonstrated its utility to transform a continuous transfer function to a discrete transfer function and vice versa.

\section{References}

[1] W. Klein. Finite Systemtheorie.TeubnerStudienbücher :Elektrotechnik. TeubnerStudienbucher Stuttgart, 1976.

[2] J. Konopacki. The frequency transformation by matrix operation and its application in iir filters design. IEEE Signal Processing Letters., 12(1):5-9, January 2005.

[3] V. Biolkova and D. Biolek. Generalized pascal matrix of first order s-z transforms. Proceedings of International Conference on Electronic Circuits and Systems ICECS, pages 929-931, 1999.

[4] Z. S. Kang. An analiticpascal matrix transform for s-to$\mathrm{z}$ domain transfer function. IEEE Signal Processing Letters, 13(10):597-600, February 2006.

[5] D. Tian-Bo, Ch. Sorowat, and D. Kobchai.Generalized pascal matrices, inverses, computations and properties using one to one rational polynomial s-z transformations.IEEE Transactions on Circuits and Systems, 55(9):368-370, October 2008.

[6] N. K. Bose. Digital Filters Theory and Applications. Elsevier Science Ltd, Amsterdam. The Netherlands, January 1986.

[7] A. Bush and D. Fielder.Simplified algebra for the bilinear and related transformations.IEEE Transactions on Audio and Electroacoustics, 21(2):127-128, April 1973.

[8] ChooYouseok and Gin KyuChoi. On pascal matrix for transformation low-pass analog filter to band-pass digital filter. IEEE SignalProcessingLetters, 16(2):878, February 2009. 


\section{Authors' Biographies}

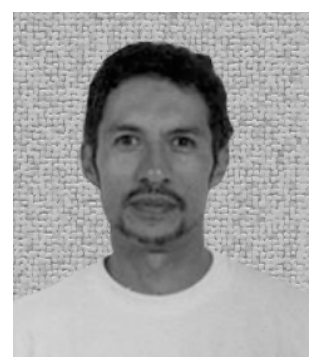

\section{Francisco Javier GARCÍA-UGALDE}

Francisco García-Ugalde obtained his bachelor's degree in electronics and electrical system engineering in 1977 from Universidad Nacional Autónoma de México, UNAM. His Diplôme d'Ingénieur in 1980 from SUPELEC, France, and his Ph.D. degree in information processing in 1982 from Université de Rennes I, France. Since 1983, he has been a full-time professor at UNAM, at the Faculty of Engineering. His current interest fields are digital filter design tools, analysis and design of digital filters, image and video coding, image analysis, theory and applications of error control coding, turbo coding, applications of cryptography, parallel processing and data bases.

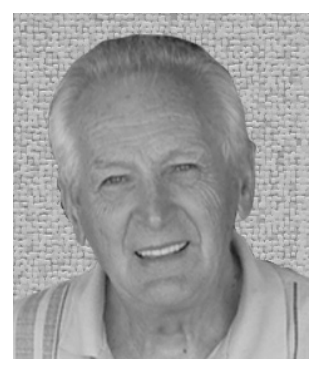

\section{Bohumil Psenicka}

Bohumil Psenicka was born in Prague in 1933. He received his C.Sc. degree from Czech Technical University in Prague in 1970. Since 1962, he has been an associate professor in the Department of Circuit Theory at the same University, and since 1993, he has been working as a C-level tenured professor at UNAM. His research interests are circuit theory, analog and digital filter design and digital signal processing.

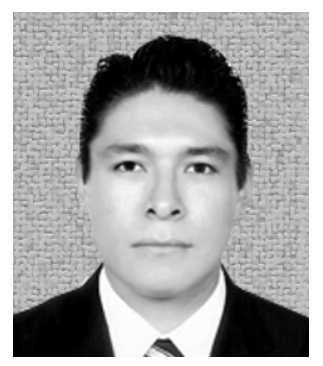

\section{Mazay Oswaldo JIMÉNEZ-SALINAS}

Mazay Jiménez-Salinas received his bachelor's degree in telecommunications engineering from UNAM in 2008. He also received his M.Sc. degree in computer science from UNAM in 2011. Since 2010, he has been working at the Faculty of Engineering at UNAM as a teaching assistant in information theory and digitals communications. His research interests include image and multi-dimensional signal processing, computational imaging, wavelets and multi-scale geometric analysis, and compressive sampling. 